\title{
Unrepeatered 64QAM over SMF-28 using Raman Amplification and Digital Backpropagation
}

\author{
Pawel Rosa ${ }^{1,2}$, Giuseppe Rizzelli ${ }^{2}$, Xiaodan Pang ${ }^{3,4}$, Oskars Ozolins ${ }^{4}$, Aleksejs Udalcovs ${ }^{4}$, Mingming Tan ${ }^{5}$, \\ Sergey Sergeyev ${ }^{5}$, Richard Schatz ${ }^{3}$, Gunnar Jacobsen ${ }^{4}$, Sergei Popov ${ }^{3}$, Juan Diego Ania-Castañón ${ }^{2}$ \\ ${ }^{1}$ National Institute of Telecommunication, Warsaw, Poland \\ ${ }^{2}$ Instituto de Óptica, IO-CSIC, Madrid, 28006, Spain \\ ${ }^{3}$ KTH Royal Institute of Technology, Isafjordsgatan 22, 16440 Kista, Sweden \\ ${ }^{4}$ RISE Acreo AB, Isafjordsgatan 22, 16440 Kista, Sweden \\ ${ }^{5}$ Aston Institute of Photonic Technologies, Aston University, Birmingham B4 7ET, UK \\ p.g.rosa@icloud.com
}

\begin{abstract}
Unrepeatered transmission over SMF-28 fibre is investigated using Raman based amplification. Experiments and simulations demonstrate a transmission up to $200 \mathrm{~km}(41 \mathrm{~dB})$ span length using 28Gbaud 64 QAM modulation employing digital back propagation in DSP.

OCIS codes: $060.1660,060.2320$.
\end{abstract}

\section{Introduction}

In fibre optic communications, quadrature amplitude modulation (QAM) with coherent detection is widely deployed due to its good balance between robustness against optical signal to noise ratio (OSNR) degradation and spectral efficiency. To maximise the transmission distance, it is necessary to maintain an acceptable OSNR through the system, which is critical while using high order modulation formats. Distributed Raman amplification ${ }^{1}$ (DRA) reduces signal decay in the fibre span leading to a higher OSNR which can allow longer reach in long-haul transmission $^{2,3}$ or longer total distance in unrepeatered systems ${ }^{4,5}$. Ultra-long Raman fibre laser (URFL) ${ }^{6}$ based amplification can further reduce the signal power variation in the fibre and has proved advantageous in comparison with EDFA only based systems ${ }^{7}$. In this paper, we experimentally investigate the performances of a single channel 28GBaud 64-QAM transmission with coherent detection over different Raman amplification schemes. The results show the benefits of employing digital back propagation (DBP) in an unrepeatered SMF-28 span without remote optically-pumped amplifier (ROPA), large effective or ultra-low loss fibre. With the reference to J. C. S. S. Januário et al. ${ }^{8}$ where authors presented unrepeatered transmission up to $370 \mathrm{~km}$ using combination of low loss fibers, forward and backward ROPA combined with amplification map optimization, theoretically, we could extend our achievable distance from $200 \mathrm{~km}(41 \mathrm{~dB})$ up to to $242 \mathrm{~km}$ employing only low-loss fibre $(0.169 \mathrm{db} / \mathrm{km})^{8}$ without forward and backward ROPA using simple design of Raman amplifiers. These are first order counter-pumped, hybrid dual order (both first and second order) and URFL based amplification with $2^{\text {nd }}$ order counter pumping.

\section{Experimental setup}

Figure 1 represents the experimental setup for a 28 Gbaud Nyquist-shaped optical 64-QAM signal. The transmitter consists of two synchronized $50 \mathrm{GSa} / \mathrm{s}$ arbitrary waveform generator (AWG), optical IQ modulator and an external cavity laser (ECL) laser having less than $100 \mathrm{kHz}$ linewidth (LW). A pseudo-random bit sequence with a word length of $2^{15}-1$ (PRBS15) is generated and Gray mapped to generate a 28Gbaud 64QAM signal followed by Nyquist pulse shaping having a 0.15 roll off factor. The sequence is then resampled to match the sampling rate of the AWGs. At the output of IQ modulator optical signal is amplified using an erbium doped amplifier (EDFA). Then signal is fed into preamplifier and band-pass filtered followed by the coherent receiver with an integrated local oscillator laser having less than $100 \mathrm{kHz} \mathrm{LW}$. A digital storage oscilloscope (DSO, $80 \mathrm{GSa} / \mathrm{s}, 33 \mathrm{GHz}$ ) is used to convert the signal into digital domain with offline demodulation.

The transmission fibre used in the experiment was standard SMF-28 with approximately $0.2 \mathrm{~dB} / \mathrm{km} \mathrm{loss}$. The measured loss in $160 \mathrm{~km}(4 \times 40 \mathrm{~km}), 200 \mathrm{~km}(5 \times 40 \mathrm{~km})$ and $240 \mathrm{~km}(6 \times 40 \mathrm{~km})$ link including splices was $33.6 \mathrm{~dB}$, $41 \mathrm{~dB}$ and $52.7 \mathrm{~dB}$, respectively. The loss from forward and backward WDM was $0.6 \mathrm{~dB}$ and $0.8 \mathrm{~dB}$, respectively. In unrepeatered $160 \mathrm{~km}$ and $200 \mathrm{~km}$ transmissions, a first order distributed backward pumped (BP) Raman laser based amplification with pump centred at $1455 \mathrm{~nm}$ was sufficient to achieve the BER below the soft FEC. However, given the strong OSNR constraints of the system at higher distance links, in a $240 \mathrm{~km}$ experiment we used second order hybrid dual/URFL bidirectional Raman pumping scheme with the highly depolarised forward and backward pumps centered at $1366 \mathrm{~nm}$. The Random distributed feedback (rDFB) lasing in the forward direction was formed due to reflected Stokes-shifted light by a high reflectivity (95\%) FBG centred at $1455 \mathrm{~nm}$ with a $0.5 \mathrm{~nm}$ bandwidth. The backward pumping was formed by two Raman pumps at $1366 \mathrm{~nm}$ and $1455 \mathrm{~nm}$. To combine and demultiplex 
Raman pumps and the signal, we used $1 \times 3 \mathrm{WDM}$ couplers in the beginning and at the end of the span. In all setups, there was an EDFA implemented before and after the transmission line.

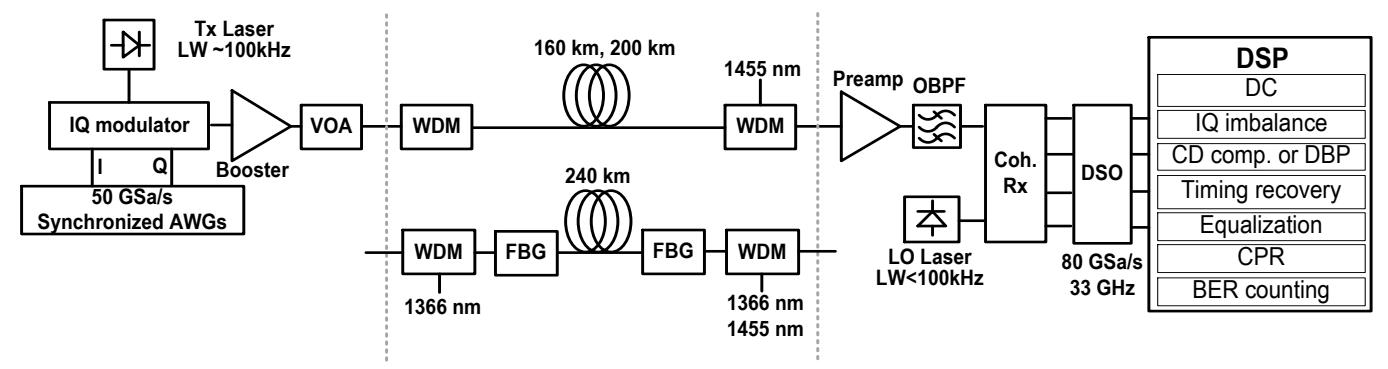

Fig. 1. Schematic design of 64 QAM experiment setup with distributed Raman amplifiers

Digital post-processing includes DBP realized by fixed step algorithm ${ }^{9}$ followed by resampling to 1 samples per symbol. A multi-modulus algorithm (MMA) equalizer is applied to compensate for linear polarization effects and finally a filtered blind phase search (F-BPS) ${ }^{10}$ for carrier frequency and phase recovery followed by error counting.

\section{Transmission results and discussions}

The constrains due to hardware limitations, the error free back to back performance of the 64 QAM transmitter was not possible. The optimal performance versus OSNR is shown in Fig. 2.

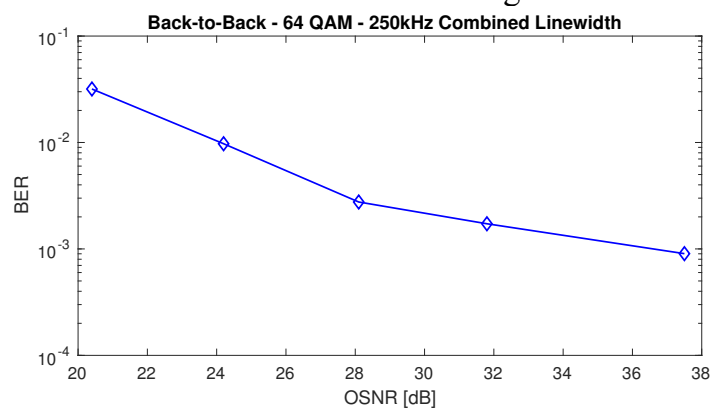

Fig. 2. BER back to back performance of a 64 QAM transmitter versus different OSNR.

The bit error rate (BER) results using first order backward Raman amplification with different input powers (I/P) over $160 \mathrm{~km}$ and $200 \mathrm{~km}$ fibre are shown in Fig. 3. In both experiments, the best BER result improvement using DBP are for the highest input powers due to higher nonlinear penalties in the beginning of the span. We may notice that there is no significant difference in the DBP improvement using higher backward Raman pump powers, which indicates that the BER improvement was noise limited. The maximum span length in the unrepeatered transmission experiment with average BER (based on 10 consecutive measurements) below the soft FEC limit $(1.9 \times 10-2)$ was $200 \mathrm{~km}$ as shown in Fig. 3.
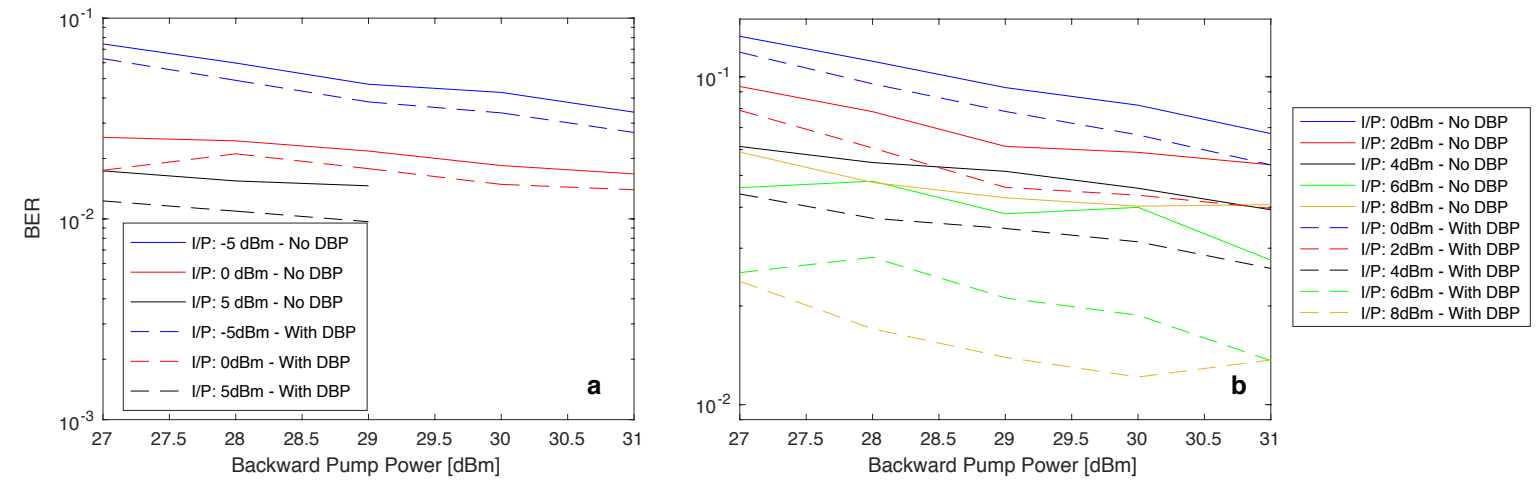

Fig. 3. BER vs BP powers for different input powers (I/P) in $160 \mathrm{~km}$ (a) and $200 \mathrm{~km}$ (b) experiment with (dashed) and without (solid) DBP. Although higher order amplification is proven ${ }^{3-5}$ to be superior in long-haul and unrepeatered transmissions, we could not achieve the BER performance that was below FEC in a $240 \mathrm{~km}$ experiment using second order 
bidirectional Raman pumping. Based on previous unrepeatered experiments using lower order modulation formats $\left(\mathrm{QPSK}^{5}\right.$ and 16QAM ${ }^{11)}$ using proposed amplification scheme, we believe that with improved back-to-back performance a successful $240 \mathrm{~km}$ SMF-28 transmission with BER below FEC is achievable. The distance could be improved considerably using low loss fibers.

Here, out of two amplification schemes that was dual order Raman with the first order backward pump at $1455 \mathrm{~nm}$ and second order at $1366 \mathrm{~nm}$ and URFL, we only present best results that were achieved with URFL configuration. The results of a $240 \mathrm{~km}$ transmission with and without DBP for different launch and total pump powers are shown in Fig. 4. We can notice that similarly to previous transmission experiments, the highest BER improvement employing DBP is for the signal with the highest launch power (yellow and green curves in Fig. 3 and Fig. 4).

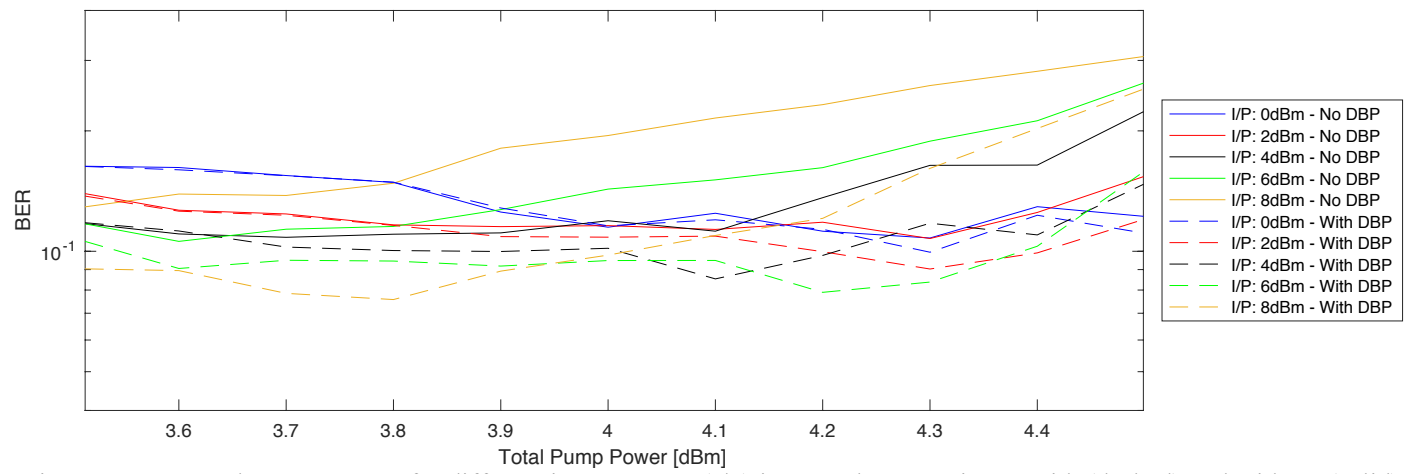

Fig. 4. BER vs total pump powers for different input powers (I/P) in a $240 \mathrm{~km}$ experiment with (dashed) and without (solid) DBP.

\section{Conclusion}

Unrepeatered transmission of a 28 Gbaud Nyquist-shaped optical 64-QAM signal up to $200 \mathrm{~km}$ has been experimentally demonstrated. Taking into account the loss of SMF-28 fiber, theoretically the distance could be extended with low loss fiber with using simple random DFB fibre laser based Raman amplification with a single pump wavelength that is compatible with both direct detection ${ }^{4}$, and advanced coherent modulation ${ }^{5}$, which means that our proposed setup could be readily used to upgrade existing installed standard single mode fibre links.

This work was funded by MSCA IF SIMFREE (748767), Swedish SRA ICT-The Next Generation project, VR project PHASE (2016-04510); the People Programme of the European Union FP7 under grant (608099) and project GRIFFON (324391); Spanish MINECO grant TEC2015-71127-C2; Comunidad de Madrid grant S2013/MIT-2790SINFOTON-CM, the Celtic-Plus sub-project SENDATE-FICUS funded by Vinnova and UK EPSRC programme grant UNLOC (EP/J017582/1). We thank Z. Sun and L. Zhang for providing FBGs.

\section{References}

[1] J. Bromage, "Raman Amplification for Fiber Communications Systems," J. Lightwave Technol., 22(1), 79-93 (2004).

[2] W. S. Pelouch, "Raman Amplification: an Enabling Technology for High-Capacity, Long-Haul Transmission," in Optical Fiber Communication Conference, OSA Technical Digest (online) (Optical Society of America, 2015), paper. W1C.1

[3] M. Tan, P. Rosa, S. Thai Le, I. D. Phillips, and P. Harper, "Evaluation of 100G DP-QPSK long-haul transmission performance using second order co-pumped Raman laser based amplification," Opt. Express 23(17), 22181-22189 (2015).

[4] P. Rosa, J. D. Ania-Castañón, and P. Harper, "Unrepeatered DPSK transmission over 360 km SMF-28 fibre using URFL based amplification," Opt. Express 22(8), 9687-9692 (2014).

[5] P. Rosa, M. Tan, S. T. Le, I. D. Philips, J. D. Ania-Castañón, S. Sygletos, and P. Harper, "Unrepeatered DP- QPSK transmission over 352.8 km SMF using random DFB fiber laser amplification" Phot. Tech. Lett. 27(11), 1041-1135 (2015).

[6] J. D. Ania-Castañón, "Quasi-lossless transmission using second-order Raman amplification and fibre Bragg gratings," Opt. Express 12(19), 4372-4377 (2004).

[7] J. D. Downie, J. Hurley, D. Pikula, S. Ten, and C. Towery, "Study of EDFA and Raman system transmission reach with 256 Gb/s PM16QAM signals over three optical fibers with $100 \mathrm{~km}$ spans" Opt. Express 21(14), 17372-17378 (2013).

[8] J. C. S. S. Januário, S. M. Rossi, S. M. Ranzini, V. E. Parahyba, V. N. Rozental, A. L. N. Souza, A. C. Bordonalli, J. R. F. Oliveira, J. D. Reis, "Unrepeatered Transmission of 10×400G over $370 \mathrm{~km}$ via Amplification Map Optimization" Phot. Tech. Lett. 28(20), 1041-1135 (2016).

[9] O. V. Sinkin et al., "Optimization of the split-step Fourier method in modeling optical-fiber communications systems," J. Lightwave Technol., Vol. 21, no. 1, p. 61 (2003).

[10] J. Rodrigo Navarro et al., "High Performance and Low Complexity Carrier Phase Recovery Schemes for 64-QAM Coherent Optical Systems," Proc. OFC, W2A.53, Los Angeles (2017).

[11] L. Galdino, M. Tan, D. Lavery, P. Rosa, R. Maher, I. D. Phillips, , J. D. Ania-Castañón, P. Harper, R. I. Killey, B. C. Thomsen, S. Makovejs, and P. Bayvel "Unrepeatered Nyquist PDM-16QAM transmission over $364 \mathrm{~km}$ using Raman amplification and multi-channel digital back-propagation," Optics Letters 40(13), 3025-3028, (2015). 